\title{
Ten years experience of syndromic surveillance for civil and military public health, France, 2004-2014
}

C Caserio-Schönemann (c.caserio-schonemann@invs.sante.fr) ${ }^{1}$, J B Meynard²

1. French Institute for Public Health Surveillance, Department of Coordination of Alerts and Regional Offices, Saint-Maurice, France

2. French Center for Epidemiology and Public Health for the French Armed Forces, Marseille, France

Citation style for this article:

Caserio-Schönemann C, Meynard JB. Ten years experience of syndromic surveillance for civil and military public health, France, 2004-2014. Euro Surveill. 2015;20(19):pii=21126. Available online: $\mathrm{http}: / /$ www.eurosurveillance.org/ViewArticle. aspx?Articleld=21126

Article submitted on 18 March 2015 / published on 14 May 2015

Difficulties by France to identify and properly estimate the impact and consequences of the intensive 2003 heatwave, and the identification of a need for an interoperable real time medical surveillance system in the French military deployed forces, has prompted France to establish two syndromic surveillance systems. These systems, a civil syndromic surveillance system 'Surveillance sanitaire des urgences et des décès' (SurSaUD) [1] and a military syndromic surveillance system, 'Le Système d'Alerte et de Surveillance en Temps Réel' (ASTER) [2] were set up in 2004. The aim of these systems is to reinforce the early warning capacity of public health events. Syndromic surveillance, defined as the (near) real time collection, analysis, interpretation and dissemination of healthrelated data aims at detecting, monitoring and evaluating unexpected, emerging or expected public health threats and contributes to the monitoring and assessment of the impact of these [3].

After a 10-year existence, the two systems SurSaUD and ASTER are pillars of the national and international public health surveillance. In November 2014, a joint meeting was organised in Paris by the French Institute for Public Health Surveillance (InVS) and the French Center for Epidemiology and Public Health for the French Armed Forces (Centre d'Epidémiologie et de Santé Publique des Armées, CESPA), including all stakeholders of the two systems. This meeting aimed at sharing the experiences and keys to success of the two systems and to promote the exchange and discussion between the different actors towards a collaborative future. All the presentations are available on the InVS website [4].

This meeting report summarises the key points of the 10-year experience, the feedback and expectations of the different stakeholders, in particular data providers and decision makers, and presents the outlook and future collaborations at a national and international level for the two systems.
Ten years of experience

Céline Caserio-Schönemann (InVS, Saint-Maurice) presented the civil syndromic surveillance system, SurSaUD. The SurSaUD system collects morbidity data from the emergency department network 'Organisation de la surveillance coordonnée des urgences' (OSCOUR) and from the emergency association of general practitioners, SOS Médecins, whose members perform home visits. The system also collects mortality data from the civil status offices and from electronic certificates. All information is transmitted automatically on a daily basis and analysed by the InVS. This system captures information about the general population in France.

The design of the military system ASTER, as presented by Jean-Baptiste Meynard (CESPA, Marseille) is quite different from SurSaUD as patient information is collected by military physicians in all areas where forces are deployed. Mobility and adaptability to several operational contexts constitute major issues for ASTER. The system requires that all data providers are still computerised for collecting data without supplementary overload.

During the meeting, three sessions illustrated how the systems have been used for each syndromic surveillance objective. Early detection was the initial objective of these systems, however syndromic surveillance has also proven useful to identify and follow trends in seasonal epidemics or for early health impact assessment of several environmental situations (extreme weather conditions, disasters, industrial accidents) or planned events (mass gatherings, exceptional events). Early detection was illustrated by Pascal Vilain (InVS, La Reunion Island Regional Office) and Gabriel Bédubourg (CESPA, Marseille) with examples of spatio-temporal analysis of several epidemics in La Reunion Island and the detection of dengue fever in French Guyana. Situational awareness with examples based on winter seasonal epidemics (influenza, bronchiolitis, gastroenteritis), asthma and the recent chikungunya fever outbreak in the Caribbean [5] was presented by Marc Ruello 
and Vanina Bousquet (InVS, Saint-Maurice), Vincent Pommier de Santi ((Direction Interarmées du Service de santé (DIASS), French Guyana Armed Forces)) and Elise Daudens (InVS, Caribbean Regional Office). Pascal Vilain, Arnaud Mathieu (InVS, Normandy Regional Office) and Vincent Pommier de Santi presented feedback from the surveillance of several exceptional events such as natural disasters, mass gatherings or industrial accidents to illustrate the objective of health risk assessment in real time.

All the presentations concluded that a key for success of these surveillance systems is the close collaboration with all the stakeholders. Continuous communication between data providers, epidemiologists, and decision makers is required to ensure efficient public health surveillance for reactive alert and action. Two open sessions of the meeting were dedicated to exchange about the feedback and expectations of data providers and decision makers.

\section{Lessons learned from data providers and decision makers}

In both the SurSaUD and ASTER surveillance systems, computerisation is a necessary prerequisite. For robust and pertinent analyses, good quality data must be collected and there must be strong adherence by health care professionals to the system. In order to optimise adherence, the system must limit the increased workload for data providers who must provide coding information, particularly for medical diagnoses. Data comparability requires a common definition and homogeneous thesaurus by all data providers. Development and dissemination of these tools have been facilitated by regional partners, the academic community, and national federations of health professionals. Ensuring good quality data required time for networking and frequent exchanges with all data providers as showed by Leslie Banzet (InVS, Languedoc-Roussillon Regional Office) and Olivier Onde (Observatoire regional des urgences du Languedoc-Roussillon, Montpellier). Sharing expertise between health professionals and epidemiologists at a regional level but also through steering committees at a national level has been another way to improve the knowledge of strengths and weaknesses of the system and to reinforce the confidence in collected datasets and in analyses produced by epidemiologists.

Though both systems are based on voluntary participation they cover a large area. One lesson learned is the importance to know and to deal with daily challenges of the data providers. Involvement in a syndromic surveillance network imposes some new constraints for health professionals in their activity (to adapt to technical tools or to mobility conditions but also to code medical information). An added value has to be found to maintain long-term participation and motivation of data providers as reported by Christophe Leroy from Louis Mourier Hospital Emergency Department, Colombes / La Société Française de Médecine d'Urgence (French
Society of Emergency Medicine) and Pascal Chansard from SOS Médecins France, Paris. Direct or indirect benefits for health professionals encourages adherence to a heath surveillance system. Targeting professional needs and expectations is a challenge as they may differ widely among and within the different networks (private or hospital physician, junior physician or head of department, mobile or in-hospital service, civilian or military context).

An added value is the health professional network that can be activated. This human dimension of the network is as important as the data transmission and analysis. Early detection is dependent on health professionals reporting unusual events.

The last main lesson learned from decision makers was the evidence of the usefulness and relevance of both systems. Communication and discussions underline the leading position of SurSaUD and ASTER as references respectively for public health surveillance and for military health surveillance at an international level.

\section{Expectations of data providers and decision makers}

Data providers primarily expect appropriate and useful feedback from epidemiologists, enabling better anticipation for healthcare organisation and a better knowledge of profiles of patients who may require emergency facilities. Data providers underline the usefulness of feedback to administrative staff in order to adapt hospital organisation to various epidemiological situations, specifically when there is a risk of overcrowding.

Alain Cadou (Champagne-Ardennes Health Regional Agency) and Catherine Guichard (Ministry of Health, Paris) recalled that communication is a crucial issue from the decision maker point of view. Epidemiological data analysis should provide clear messages that should be transmitted to the appropriate contact person for timely decision making. The information needed by health authorities should be easily understandable and should be transmitted using the most appropriate means of communication. The baseline, i.e. what is expected in normal situation, has to be specified as well as temporal elements concerning when the situation will recover, underlined Emmanuel Angot from the Military Health Service (Service de santé des armées), Paris. Because syndromic surveillance provides timesensitive data, it is important to ensure consistency between the request of decision makers for the most up to date information and the epidemiologist's capacity to effectively assess the information. This is particularly pertinent in situations with intensive media coverage. Time for decision making is different from time for epidemiological analysis and output. A balance has to be struck between both the needs to select what is relevant to communicate, to ensure a minimum of data reliability and not to burn out forces. Health authorities can explore two approaches. One approach is to develop 'positive' communication targeted to 
media out of any heath event that aims to educate and to limit media pressure on health authorities in case of a public health event. Gilles Viudes from the Federation of Regional Observatories for Emergency Activities (Fédération des Observatoires régionaux des urgences) suggested that another approach for decision makers is to improve anticipation and preparedness with tools such as management protocols for different geographical scales using information accrued from the ten years of experience with the syndromic surveillance systems. For example, adapting regional control measures to anticipate seasonal influenza, gastroenteritis or bronchiolitis epidemics that occur every year at about the same period could be strongly relevant to reduce hospital overcrowding. These protocols would have to be widely disseminated to allow for a proper understanding of what is to be done, who needs to act and when. Health authorities should consider syndromic surveillance as a timely, sound scientific basis to better support preparedness and decision making.

All data providers and decision makers agreed that IT aspects, particularly business software solutions, are a major issue for these systems. Even if syndromic surveillance systems have been probable levers for accelerating health professional computerisation in the past, a real challenge and a recurrent request from data providers is to make software tools (especially coding tools) and applications more user-friendly and better suited to clinical practice. This is essential in order to maintain the participation of data providers and encourage the practice of coding medical information. Collaboration with software developers would be useful in order to develop effective and interoperable electronic information systems including tools to streamline procedure and to enable exchange of high quality datasets.

\section{Prospects}

Prospects for both systems deal with mutual concerns but also specific issues.

Both systems aim to increase methodological developments to enhance data analysis. Both syndromic surveillance systems now are based on huge databases with ten years worth of historical data. The implementation of robust and performant statistical methods is required in order to meet the objective of detection of the systems. From a technical point of view, additional tools have to be developed in order to adapt systems to the rapid evolution of IT technology and in order to deal with the concern of the increase in data volume and the need to develop new tools to analyse this vast amount of data. Céline Caserio-Schönemann suggested that new technological strategies can be explored such as developing free text analysis and data mining, and promoting training with the use of simulation platform as underlined by Hervé Chaudet (CESPA, Marseille).

Common goals are also geared towards long-standing international collaborations. European collaborations have been strengthened by the Triple $S$ project from 2010 to 2013 [6] led by the InVS and involving CESPA as reported by Anne Fouillet (InVS, SaintMaurice), Hervé Chaudet (CESPA, Marseille) and Anne Bronner of the French Agency for Food, Environmental and Occupational Health and Safety, Lyon (Agence nationale de sécurité sanitaire de l'alimentation, de l'environnement et du travail). The Triple $S$ project made it possible to build a European syndromic surveillance network and to share expertise. It resulted in the publication of deliverables such as the European definition of syndromic surveillance [3], guidelines for designing and implementing a syndromic surveillance system [7], and the proposal for a European strategy for syndromic surveillance [8]. At an international level, collaborations should also be reinforced through the International Society for Disease Surveillance [9]. For the ASTER system one crucial issue is to enter fully into a process of industrialisation and deployment at an international level in order to build the model for the future North Atlantic Treaty Organization syndromic surveillance system as explained by Benjamin Queyriaux (Deployment Health Surveillance Capability, Munich).

Some issues are specific to the SurSaUD system. The first is the strengthening of existing data sources. If efforts have to be done to maintain the emergency department network OSCOUR as an exhaustive network without compromising quality, special attention has to be paid to develop the use of electronic death certificates. Grégoire Rey from the French Epidemiological Center for Medical Causes of Death (Centre d'épidémiologie sur les causes médicales de décès, Le Kremlin Bicêtre), expressed his regret at the fact that at the end of 2014 only $6 \%$ of deaths were certified online. This level is insufficient to allow proper analysis for timely health surveillance and alert. Regional Health Agencies should examine this issue and promote the use of electronic death certificates in healthcare facilities. In parallel, a specific methodological issue for the syndromic surveillance team concerns building pertinent and efficient indicators based on free text analysis of the medical causes of death.

The introduction of new data sources in the surveillance system in order to have a better overview of health population is also being explored. Developing collaboration with the Emergency Medical Services (Service d'Aide Médicale Urgente) to gain in complementary and earlier information is in progress as presented by Florian Franke (InVS, South Regional Office).

\section{Conclusions}

After ten years of existence both civilian and military French syndromic surveillance systems are mature systems with fruitful experiences demonstrating the efficiency and complementarity with traditional surveillance systems. The future of syndromic surveillance is open to innovation, sharing of expertise and development of scientific synergies with data providers, syndromic surveillance partners (in human 
and veterinarian fields) and the scientific community emphasised Anne Gallay (InVS, Saint-Maurice) and Rémy Michel (CESPA, Marseille). Scientific promotion of French syndromic surveillance results will certainly help to meet the goals.

Designing and implementing a communication strategy towards stakeholders is crucial. In particular, attempts should be made to simplify information content, improve user awareness and tailor information and feedback to the needs of users in order to ensure that syndromic surveillance proves helpful for decision making in public health.

\section{Acknowledgments}

The organisers of the meeting would like to thank partners and networks involving in the SurSaUD and ASTER syndromic surveillance systems, the meeting scientific committee, both InVS and CESPA syndromic surveillance and meeting organization teams and all the attendees of the meeting. Particular thanks are addressed to Anne Fouillet (InVS) and Gabriel Bédubourg (CESPA) for widely contributing to the preparation of this meeting report and to InVS colleagues for writing a summary of the different sessions and discussions: Pascal Chaud, Marlène Faisant, Florian Franke, Gaëlle Gault, Magali Lainé, Laure Meurice, Jean-Rodrigue NDong.

\section{Conflict of interest}

None declared.

\section{Authors' contributions}

Both authors contributed to the writing of the meeting report.

\section{References}

1. Caserio-Schönemann C, Bousquet V, Fouillet A, Henry V for the SurSaUD team. Le système de surveillance syndromique SurSaUD. [The French syndromic surveillance system SUrSaUD]. Bull Epidemiol Hebd (Paris). 2014 January;3-4 (22):38-44. French. Available from: http://www.invs.sante.fr/ beh/2014/3-4/index.html

2. Meynard JB, Chaudet H, Texier G, Ardillon V, Ravachol F, Deparis X, et al. Value of syndromic surveillance within the Armed Forces for early warning during a dengue fever outbreak in French Guiana in 2006. BMC Med Inform Decis Mak. 2008;2(8):29. 10.1186/1472-6947-8-29

3. Triple S Project. Assessment of syndromic surveillance in Europe. Lancet. 2011;378(9806):1833-4. http://dx.doi org/10.1016/S0140-6736(11)60834-9

4. Institut de Veille Sanitaire/ Centre d'épidémiologie et de santé publique des armées (InVS/CESPA). 1ères Journées Scientifiques SurSaUD/ASTER. [First scientific days SurSaUD/ ASTER]. Meeting agenda 20-21 November 2014. French. Available from: http://www.invs.sante.fr/Actualites/ Agenda/1eres-Journees-Scientifiques-SurSaUD-R-ASTER

5. Van Bortel W, Dorleans F, Rosine J, Blateau A, Rousset D, Matheus S, et al. Chikungunya outbreak in the Caribbean region, December 2013 to March 2014, and the significance for Europe. Euro Surveill. 2014;19(13):pii=20759. http://dx.doi. org/10.2807/1560-7917.es2014.19.13.20759

6. Fouillet A, Medina S, Medeiros H, Sala Soler M, Dupuy C et al. La surveillance syndromique en Europe : le projet européen Triple-S. [Syndromic surveillance in Europe : the European Triple-S Project ]. Bull Epidemiol Hebd (Paris). 2014 January; 3-4(22):75-80. French. Available from: http://www.invs.sante. $\mathrm{fr} / \mathrm{beh} / 2014 / 3-4 /$ index.html

7. Triple-S consortium. Guidelines for designing and implementing a syndromic surveillance system. Deliverable
8, Work Package 6; 2013. Available from: http://www. syndromicsurveillance.eu/Triple-S_guidelines.pdf

8. Triple-S consortium. Proposal for a European syndromic surveillance strategy. Deliverable 9, Work Package 6; 2013. Available from: http://www.syndromicsurveillance.eu/ Triple-S_proposal.pdf

9. International Society for Disease Surveillance. [Accessed 13 May 2015]. Available from: http://www.syndromic.org/ 